\title{
Mudanças nomenclaturais no gênero TaChigali Aubl. (Leguminosae - Caesalpinioideae) no Brasil
}

\author{
Luciana Fernandes Gomes da Silva ${ }^{1} \&$ Haroldo Cavalcante de Lima ${ }^{2}$
}

\begin{abstract}
RESUMO
(Mudanças nomenclaturais no gênero Tachigali Aubl. (Leguminosae - Caesalpinioideae) no Brasil) Estudos recentes sobre sistemática de leguminosas demonstraram que Sclerolobium deve ser tratado como sinônimo de Tachigali. Neste trabalho são apresentadas as novas combinações para 11 táxons ocorrentes no Brasil: $T$. beaurepairei (Harms) L. G. Silva \& H. C. Lima, T. densiflora (Benth.) L. G. Silva \& H. C. Lima, T. eriopetala (Ducke) L. G. Silva \& H. C. Lima, T. friburgensis (Harms) L. G. Silva \& H. C. Lima, T. froesii (Pires) L. G. Silva \& H. C. Lima, T. goeldiana (Huber) L. G. Silva \& H. C. Lima, T. leiocalyx (Ducke) L. G. Silva \& H. C. Lima, T. macropetala (Ducke) L. G. Silva \& H. C. Lima, T. prancei (H. S. Irwin \& Arroyo) L. G. Silva \& H. C. Lima, T. subullata (Ducke) L. G. Silva \& H. C. Lima e T. urbaniana (Hams) L. G. Silva \& H. C. Lima. Foi necessário propor um novo nome ( $T$. vulgaris L. G. Silva \& H. C. Lima) e dois novos sinônimos (S. glaziovii Taub. $=$ T. denudata e S. striatum Dwyer = T. pilgeriana). São também fornecidos dados sobre os tipos e os nomes corretos de algumas espécies ocorrentes no Sudeste do Brasil: T. aurea Tul., T. denudata (Vogel) Oliveira-Filho, T. duckei (Dwyer) Oliveira-Filho, T. pilgeriana (Harms) Oliveira-Filho, T. rubiginosa (Tul.) Oliveira-Filho e T. subvelutina (Benth.) Oliveira-Filho. Palavras-chave: Sclerolobium, taxonomia, nomenclatura, leguminosa, Caesalpinieae.
\end{abstract}

\begin{abstract}
(Nomenclatural changes in the genus Tachigali Aubl. (Leguminosae - Caesalpinioideae) in Brazil) Recent studies on legume systematics have demonstrated that Sclerolobium should be treated as a synonym of Tachigali. In this work, eleven new combinations are presented: T beaurepairei (Harms) L. G. Silva \& H. C. Lima, T. densiflora (Benth.) L. G. Silva \& H. C. Lima, T. eriopetala (Ducke) L. G. Silva \& H. C. Lima, T. friburgensis (Harms) L. G. Silva \& H. C. Lima, T. froesii (Pires) L. G. Silva \& H. C. Lima, T. goeldiana (Huber) L. G. Silva \& H. C. Lima, T. leiocalyx (Ducke) L. G. Silva \& H. C. Lima, T. macropetala (Ducke) L. G. Silva \& H. C. Lima, T. prancei (H. S. Irwin \& Arroyo) L. G. Silva \& H. C. Lima, T. subullata (Ducke) L. G. Silva \& H. C. Lima and T. urbaniana (Hams) L. G. Silva \& H. C. Lima. A new name (T. vulgaris L. G. Silva \& H. C. Lima) and two new synonyms are proposed $(S$. glaziovii Taub. $=T$. denudata e $S$. striatum Dwyer $=T$. pilgeriana). Correct names and type information for other six species that occur in Southeastern Brazil are also provided (T. aurea Tul., T. denudata (Vogel) Oliveira-Filho, T. duckei (Dwyer) Oliveira-Filho, T. pilgeriana (Harms) Oliveira-Filho, T. rubiginosa (Tul.) Oliveira-Filho e T. subvelutina (Benth.) Oliveira-Filho).
\end{abstract}

Key words: Sclerolobium, taxonomy, nomenclature, legume, Caesalpinieae.

\section{INTRODUÇÃO}

Entre os gêneros de leguminosas arbóreas neotropicais, Tachigali e Sclerolobium destacam-se pela elevada riqueza de espécies e pela abundância em ambientes de margens de rios e em formações florestais em regeneração (L. F. G. Silva \& H. C. Lima, dados não publicados). Embora nas últimas revisões (Dwyer 1954, 1957) tenham sido considerados distintos e reconhecidas respectivamente $23 \mathrm{e}$ 34 espécies, a delimitação genérica mostrava- se ainda bastante imprecisa. Estudos mais recentes, principalmente investigando a anatomia do lenho (Barreta-Kuipers 1981), a morfologia dos grãos de pólen (Graham \& Barker 1981) e caracteres macromoleculares (Haston et al. 2003, 2005), além da constatação de espécies com caracteres intermediários ou comuns entre os dois gêneros, apoiaram a proposição de alguns autores de considerá-los sinônimos (Zarucchi \& Herendeen 1993; Pipoly 1995; Lewis 2005).

Artigo recebido em 09/2006. Aceito para publicação em 05/2007.

${ }^{1}$ Mestranda da Escola Nacional de Botânica Tropical/JBRJ. lufgdasilva@ig.com.br

${ }^{2}$ Instituto de Pesquisas Jardim Botânico do Rio de Janeiro, Rua Pacheco Leão 915, Jardim Botânico, 22460-030, Rio de Janeiro, RJ, Brasil. hlima@jbrj.gov.br 
Os estudos comparativos sobre a morfologia de espécies ocorrentes no Brasil (L. F. G. Silva \& H. C. Lima, dados não publicados) também evidenciaram a grande semelhança entre os dois gêneros, fortalecendo a proposta de incluir Sclerolobium como sinônimo de Tachigali. Neste contexto, foi aceita a expansão do conceito genérico de Tachigali para incluir espécies com hipanto cupular com estípite aderido ao fundo. Estes resultados também são apoiados por estudos recentes ainda inéditos com base em dados moleculares, que sustentam a monofilia no gênero com esta circunscrição (V. H. Maia, dados não publicados).

O objetivo do presente trabalho é divulgar os resultados da revisão nomenclatural efetuada em Tachigali, formalizando as mudanças necessárias para estabelecer os nomes corretos de espécies ocorrentes no Brasil. São ainda fornecidos dados complementares sobre os tipos de táxons da Região Sudeste do Brasil, principalmente das espécies recentemente transferidas para Tachigali por Oliveira-Filho (2006).

\section{Material e Métodos}

A revisão nomenclatural foi realizada com base na lista de espécies ocorrentes no Brasil, que foi preparada a partir do inventário na literatura e de herbários. Foram consultados os principais herbários brasileiros (CEPEC, CVRD, GUA, IAN, IBGE, INPA, MBM, MBML, MG, R, RB, RBR, RFA, SP, UB, UEC, VIC) - siglas citadas conforme Holmgren et al. 1990. Um estudo complementar constou do exame do material tipo da maioria das espécies ou de fotografias dos mesmos, que foram assinalados no texto com sinal de exclamação (!).

$\mathrm{Na}$ avaliação da tipificação dos táxons, sempre que possível, foram indicados os holótipos e isótipos. Entretanto, face à impossibilidade de examinar todas as coleções síntipicas, não foram escolhidos os lectótipos das espécies.

\section{Resultados e Discussão}

1. Tachigali aurea Tul., Arch. Mus. Hist. Nat. 4: 169. 1844. Tipo: "Cujaba Brasiliae austro-occidentalis", 1841, A. L. P. Silva Manso (Mart. Herb. Fl. Bras. ${ }^{\circ} 1148$ ), (lectótipo B; isolectótipos F, NY, US; foto lectótipo-RB!).

Sclerolobium aureum (Tul.) Benth., Fl. bras. 15(2): 51. 1870.

Sclerolobium aureum var. velutinum Benth., Fl. bras. 15(2): 51. 1870. Tipo: BRASIL. "in campis siccis ad Rio Coxim prov. Mato Grosso", L. Riedel (holótipo K; isótipo F).

Tulasne (1844), sem aplicação formal de nomes infra-específicos, descreveu duas formas em T. aurea, citando os materiais Claussen (Cat. Herb. Bras. Mus. $\mathrm{n}^{\circ}$ 965) e Blanchet 3080 para a forma $\alpha$ e Mart. Herb. Fl. Bras. no 1148 para a forma $\beta$. Posteriormente, Dwyer (1957) designou este último material como o lectótipo desta espécie.

A variação na morfologia foliar, principalmente o número de folíolos e indumento, foi utilizada por Bentham (1870) para descrever Sclerolobium aureum var. velutinum. No presente estudo, T. aurea é reconhecida em seu sentido amplo e aceita a proposta de Dwyer (1957) que considerou esta variedade um sinônimo.

\section{Tachigali beaurepairei (Harms) L. G. Silva} \& H. C. Lima, comb. nov.

Sclerolobium beaurepairei Harms, Bot. Jahrb. Syst. 33(72): 23. 1903. Tipo: BRASIL. RIO DE JANEIRO: "Alto Macahé de Nova Friburgo", XI.1890, A. F. Glaziou 18206 (síntipo B; isosíntipos F, NY, P, R; foto síntipo-RB!); idem, 1891, A. F. Glaziou 19879 (síntipo B; isosíntipos F, NY, P; foto síntipo-RB!); idem, 4.XII.1892, A. F. Glaziou 20286 (sintipo B; isosíntipos F, K, MO, NY, P, R, RB!, US). 
3. Tachigali densiflora (Benth.) L. G. Silva \& H. C. Lima, comb. nov.

Sclerolobium densiflorum Benth., Fl. bras. 15(2): 51. 1870. Tipo: BRASIL. "In parte australi prov. Bahiensis", J. S. Blanchet 3206A (holótipo K; isótipo MO).

4. Tachigali denudata (Vog.) Oliveira-Filho, Cat. Árvores Nativas Minas Gerais 140. 2006.

Sclerolobium denudatum Vog., Linnaea 11: 396. 1837. Tipo: "in Brasil. Merid.", $F$. Sellow (holótipo B; isótipos F, K, MO, US; foto holótipo-RB!).

Sclerolobium glaziovii Taub., Flora 75(50):80. 1892. Tipo: BRASIL. RIO DE JANEIRO: "Floresta da Tijuca", 2.IX.1882, A. F. Glaziou 13735 (holótipo B; isótipos F, MO, K, P, RB!, US), syn. nov.

$\mathrm{Na}$ análise do rico material botânico atualmente disponível, além de estudos em exemplares no campo, foi observada uma considerável uniformidade nos caracteres florais e grande variação na presença de indumento na face abaxial dos folíolos, desde densamente cano-pubescentes até glabrescente. É importante salientar que esta variação na densidade e distribuição do indumento foi constatada dentro do mesmo indivíduo. A partir do resultado desta análise foi possível constatar que S. glaziovii deve ser colocada em sinônimo de $T$. denudata.

5. Tachigali duckei (Dwyer) Oliveira-Filho, Cat. Árvores Nativas Minas Gerais 140. 2006.

Sclerolobium duckei Dwyer, Lloydia 20(2): 109. 1957. Tipo: BRASIL. RIO DE JANEIRO: "Itatiaia", Parque Nacional, Lote Hansen, 1.X.1940, W. D. Barros 48 (holótipo $\mathrm{R}$; isótipo RB!). No protólogo foi citado "Barries" como coletor do material tipo.

6. Tachigali eriopetala (Ducke) L. G. Silva \& H. C. Lima, comb. nov.

Sclerolobium eriopetalum Ducke, Arch. Inst. Biol. Veg. 2(1): 41. 1935. Tipo: BRASIL.
AMAZONAS: Manaus, Rio Tarumã, 8.VII.1933, A. Ducke (holótipo RB 24296!; isótipos $\mathrm{F}, \mathrm{K}, \mathrm{P}, \mathrm{MG}$ ).

7. Tachigali friburgensis (Harms) L. G. Silva \& H. C. Lima, comb. nov.

Sclerolobium friburgense Harms, Repert. Spec. Nov. Regni Veg. 24: 211. 1928. Tipos: BRASIL. RIO DE JANEIRO: "Alto Macahé de Nova Friburgo", 26.XII.1881, A. F. Glaziou 13734 (sintipo P, isosíntipo R); idem, I.1892, A. F. Glaziou 19059 (síntipo $\mathrm{B}$; isosíntipos F, K, MO, P, R, US; foto síntipo-RB!).

8. Tachigali froesii (Pires) L. G. Silva \& H. C. Lima, comb. nov.

Sclerolobium froesii Pires, Bol. Tecn. Inst. Agron. N. 38: 23-24, pl.6,11,f.1. 1960. Tipo: BRASIL. AMAZONAS: Rio Canumã, região do rio Madeira, 5.XI.1957, R. L. Froes 33744 (holótipo MG; isótipo RB!).

9. Tachigali goeldiana (Huber) L. G. Silva \& H. C. Lima, comb. nov.

Sclerolobium goeldianum Huber, Bol. Mus. Paraense Hist. Nat. 6: 78. 1910. Tipo: BRASIL. PARÁ: Rio Capim, 15.VII.1897, J. Huber s.n. (holótipo MG 692; isótipos $\mathrm{F}, \mathrm{MO}, \mathrm{NY}, \mathrm{RB}$ !).

10. Tachigali leiocalyx (Ducke) L. G. Silva \& H. C. Lima, comb. nov.

Sclerolobium leiocalyx Ducke, Bol. Tecn. Inst. Agron. N. 2: 19. 1944. Tipo: BRASIL. AMAZONAS: São Paulo de Olivença, 15.X.1942, A. Ducke 1028 (holótipo RB!; isótipos $\mathrm{K}, \mathrm{MO}, \mathrm{NY}$ ).

11. Tachigali macropetala (Ducke) L. G. Silva \& H. C. Lima, comb. nov.

Sclerolobium macropetalum Ducke, Arch. Inst. Biol. Veg. 2(1):41.1935. Tipo: BRASIL. AMAZONAS: Rio Negro, Curicuriary, 14.X.1932, A. Ducke s.n. (holótipo RB 23328!, isótipos: F, NY, U). 
12. Tachigali pilgeriana (Harms) OliveiraFilho, Cat. Árvores Nativas Minas Gerais 140. 2006.

Sclerolobium pilgerianum Harms, Bot. Jahrb. Syst. 33(72): 24. 1903. Tipo: BRASIL. RIO DE JANEIRO: "Petrópolis, Caxambú", III.1886, A. F. Glaziou 15933 (holótipo B, isótipos $\mathrm{F}, \mathrm{MO}, \mathrm{P}$, US; foto holótipo-RB!)

Sclerolobium striatum Dwyer, Lloydia 20(2): 87. 1957. Tipo: BRASIL. RIO DE JANEIRO: "Botanical Garden", 7.II.1948, Pessoal do Jardim Botânico s.n. (holótipo MO; isótipos RFA!, RB 61518!), syn. nov.

A semelhança nos caracteres florais, principalmente no que diz respeito ao indumento das pétalas e tamanho do pedicelo, e a grande variação na morfologia das folhas sustentam a proposição de incluir $S$. striatum como sinônimo de T. pilgeriana.

13. Tachigali prancei $(\mathrm{H}$. S. Irwin \& M. T. Arroyo) L. G. Silva \& H. C. Lima, comb. nov. Sclerolobium prancei $\mathrm{H}$. S. Irwin \& Arroyo, Brittonia 26(3): 268. 1974. Tipo: BRASIL. RONDÔNIA: Serra dos Três Irmãos, north bank of rio Madeira, opposite Mutumparaná, 3.VII.1968, G. T. Prance et al. 5524 (holótipo NY; isótipos INPA, MG, RB!).

14. Tachigali rubiginosa (Mart. ex Tul.) Oliveira-Filho, Cat. Árvores Nativas Minas Gerais 141. 2006.

Sclerolobium rubiginosum Mart. ex Tul., Arch. Mus. Hist. Nat. 4: 123. 1844. Tipo: "in sylvis prope Cujaba Brasiliae meridionalis", 1841, A. L. P. Silva Manso (Mart. Herb. Fl. Bras. n¹147), (holótipo P; isótipos: $\mathrm{B}, \mathrm{F}, \mathrm{NY}, \mathrm{W}$; foto isótipo NY!).

Sclerolobium paniculatum var. rubiginosum (Mart. ex Tul.) Benth., Fl. bras. 15(2): 47.1870.

15. Tachigali subullata (Ducke) L. G. Silva \& H. C. Lima, comb. nov.

Sclerolobium subullatum Ducke, Arch. Inst. Biol. Veg. 2: 42. 1935. Tipo: BRASIL.
AMAZONAS: Esperança, boca do Javari, 19.IX.1931, A. Ducke s.n. (holótipo RB 24298!; isótipos K, P).

16. Tachigali subvelutina (Benth.) OliveiraFilho, Cat. Árvores Nativas Minas Gerais 141. 2006.

Sclerolobium paniculatum var. subvelutinum Benth., Fl. bras. 15(2): 48. 1870. Tipos: BRASIL. "ad fl. Paranahyba, prov. Goyaz”, W. J. Burchell 793A (holótipo K; isótipos: NY, P; foto isótipo NY - RB!).

17. Tachigali urbaniana (Hams) L. G. Silva \& H. C. Lima, comb.nov.

Sclerolobium urbanianum Harms, Bot. Jahrb. Syst. 33 (72): 23. 1903. Tipo. BRASIL. RIO DE JANEIRO, Serra do Tinguá, Rio D'Ouro, 24.VIII.1879, A. F. Glaziou 10683 (holótipo B; isótipos F, K, MO, R; foto holótipo-RB!).

18. Tachigali vulgaris L. G. Silva \& H. C. Lima, nom. nov.

Sclerolobium paniculatum Vogel, Linnaea 11: 397. 1837. Tipo: BRASIL. "in regione Cujabá prov. Matto-Grosso in sylvis", A. L. P. Silva Manso \& J. Lhotsky (holótipo $\mathrm{B}$; isótipo $\mathrm{MO}$; foto isótipo-RB!).

Um novo nome esta sendo proposto para a espécie devido à existência de Tachigali paniculata Aubl. Na circunscrição de Sclerolobium paniculatum Vog. proposta por Dwyer (1957), três táxons infraespecíficos foram reconhecidos para o Brasil: S. paniculatum var. paniculatum, S. paniculatum var. subvelutinum Benth. e S. paniculatum var. rubiginosum (Mart. ex Tul.) Benth. Em estudo recente sobre as árvores nativas de Minas Gerais, OliveiraFilho (2006) transferiu as duas últimas variedades para o gênero Tachigali, considerando as mesmas na categoria de espécie: T. rubiginosa (Mart. ex Tul.) Oliveira-Filho e T. subvelutina (Benth.) Oliveira-Filho. 


\section{Agradecimentos}

Aos curadores dos herbários brasileiros pelo empréstimo do material e aos curadores dos herbários $\mathrm{B}, \mathrm{K}, \mathrm{MO}$ e $\mathrm{P}$ pelo envio das fotografias dos tipos. Ao Dr. Jorge Fontella Pereira, Museu Nacional/UFRJ, pelos valiosas sugestões. Ao Vitor Hugo dos Santos Gomes Maia, pós-graduando da Escola Nacional de Botânica Tropical (ENBT/JBRJ), pelo fornecimento dos dados inéditos do projeto de filogenia molecular do grupo Sclerolobium. Ao Programa Mata Atlântica (Convênio 610.4.025.02.3 - JBRJ/PETROBRAS) pelo apoio financeiro às atividades de campo.

\section{REFERÊNCIAS BIBLIOGRÁFICAS}

Barreta-Kuipers, T. 1981. Wood anatomy of Leguminosae: its relevance to taxonomy In: Polhill, R. M. \& Raven, P. H. (eds.). Advances Legume Systematics Part 2. Royal Botanic Gardens, Kew. Pp. 677-706.

Bentham, G. 1870. Sclerolobieae. In: Martius, C. F. P. von; Eichler, A. W. \& Urban, I. Flora brasiliensis. F. Fleischer, Lipsiae, 15(2): 45-51.

Dwyer, J. D. 1954. The tropical American genus Tachigalia Aubl. (Caesalpiniaceae). Annals of the Missouri Botanical Garden 41(2): 223-260.

1957. The tropical American genus Sclerolobium Vogel (Caesalpiniaceae). Lloydia 20 (2): 68-118.

Graham, A. \& Barker, G. 1981. Palinology and tribal classification in the Caesalpinioideae. In: Polhill, R. M. \& Raven, P. H. (eds.). Advances Legume Systematics Part 2. Royal Botanic Gardens, Kew. Pp. 801-834.
Haston, E. M.; Lewis, G. P. \& Hawkins, J. A. 2003. A phylogenetic investigation of the Peltophorum group (Caesalpinieae: Leguminosae). In: Klitgaard, B. B. \& Bruneau, A. (eds.). Advances in Legume Systematics. Part 10. Royal Botanic Gardens, Kew. Pp. 149-159.

2005. A phylogenetic reappraisal of the Peltophorum group (Caesalpinieae: Leguminosae) Based on the Chloroplast trnL-F, rbcL and rpsl6 Sequence Data. American Journal of Botany 92(8): 1359-1371.

Holmgren, P. K.; Holmgren, N. H. \& Barnett, L. C. (eds.). 1990. Index Herbariorum Part I: The Herbaria of the World. 8th ed. New York Botanical Garden, New York, 693p.

Lewis, G. P. 2005. Caesalpinieae. In: Lewis, G. P.; Schrire, B., Mackinder B. \& Lock, M. (eds.). Legumes of the world. Royal Botanic Gardens, Kew. Pp. 127-161.

Oliveira-Filho, A. T. 2006. Tachigali. In: Catálogo das árvores nativas de Minas Gerais: mapeamento e inventário da flora nativa e dos reflorestamentos de Minas Gerais. Editora UFLA, Lavras. Pp. 140-141.

Pipoly, J. J. 1995. A new Tachigali (Fabaceae: Caesalpinioideae) from western Amazonia. Sida 16(3): 407-411.

Zarucchi, J. \& Herendeen, P. 1993. Tachigali (Fabaceae). In: Brako, L. \& Zarucchi, J. (eds.). Catalogue of the flowering plants and Gymnosperms of Peru. Monographs in Systematic Botany from Missouri Botanical Garden 45. Pp. 1254-1255. 